\title{
Persistence and Nonpersistence of a Nonautonomous Stochastic Mutualism System
}

\author{
Peiyan Xia, ${ }^{1,2}$ Xiaokun Zheng, ${ }^{2}$ and Daqing Jiang ${ }^{2}$ \\ ${ }^{1}$ School of Basic Sciences, Changchun University of Technology, Changchun, Jilin 130021, China \\ ${ }^{2}$ School of Mathematics and Statistics, Northeast Normal University, Changchun, Jilin 130024, China \\ Correspondence should be addressed to Daqing Jiang; daqingjiang2010@hotmail.com
}

Received 22 October 2012; Accepted 29 November 2012

Academic Editor: Jifeng Chu

Copyright (C) 2013 Peiyan Xia et al. This is an open access article distributed under the Creative Commons Attribution License, which permits unrestricted use, distribution, and reproduction in any medium, provided the original work is properly cited.

\begin{abstract}
In this paper, a two-species nonautonomous stochastic mutualism system is investigated. The intrinsic growth rates of the two species at time $t$ are estimated by $r_{i}(t)+\sigma_{i}(t) \dot{B}_{i}(t), i=1,2$, respectively. Viewing the different intensities of the noises $\sigma_{i}(t), i=1,2$ as two parameters at time $t$, we conclude that there exists a global positive solution and the $p$ th moment of the solution is bounded. We also show that the system is permanent, including stochastic permanence, persistence in mean, and asymptotic boundedness in time average. Besides, we show that the large white noise will make the system nonpersistent. Finally, we establish sufficient criteria for the global attractivity of the system.
\end{abstract}

\section{Introduction}

For more than three decades, mutualism of multispecies has attracted the attention of both mathematicians and ecologists. By definition, in a mutualism of multispecies, the interaction is beneficial for the growth of other species. LotkaVolterra mutualism systems have long been used as standard models to mathematically address questions related to this interaction. Among these, nonautonomous Lotka-Volterra mutualism models are studied by many authors, see [1-7] and references therein. The classical nonautonomous LotkaVolterra mutualism system can be expressed as follows:

$$
\begin{aligned}
& \dot{x}_{i}(t)=x_{i}(t)\left[r_{i}(t)-a_{i i}(t) x_{i}(t)+\sum_{\substack{j=1 \\
j \neq i}}^{n} a_{i j}(t) x_{j}(t)\right], \\
& i=1,2, \ldots, n,
\end{aligned}
$$

where $x_{i}(t), i=1,2, \ldots, n$ is the density of the $i$ th population at time $t, r_{i}(t)>0, i=1,2, \ldots, n$ is the intrinsic growth rate of the $i$ th population at time $t, r_{i}(t) / a_{i i}(t)>0, i=$ $1,2, \ldots, n$ is the carrying capacity at time $t$, and coefficient $a_{i j}(t)>0, i, j=1,2, \ldots, n$ describes the influence of the $j$ th population upon the $i$ th population at time $t$.
It is shown in [1] that if different conditions hold (see conditions (a)-(e) in [1]), then the solution of system (1) is bounded, permanent, extinct, and global attractive, respectively. However, when the intrinsic growth rate and coefficient $a_{i j}(t)$ are periodic, it is shown in [3] that there exists positive periodic solution and almost periodic solutions are obtained.

From another point of view, environmental noise always exists in real life. It is an interesting problem, both mathematically and biologically, to determine how the structure of the model changes under the effect of a fluctuating environment. Many authors studied the biological models with stochastic perturbation, see [8-12] and references therein. In [8] Ji et al. discussed the following two-species stochastic mutualism system

$$
\begin{aligned}
& d x_{1}(t)=x_{1}(t)\left[\left(r_{1}-a_{11} x_{1}(t)+a_{12} x_{2}(t)\right) d t+\sigma_{1} d B_{1}(t)\right], \\
& d x_{2}(t)=x_{2}(t)\left[\left(r_{2}+a_{21} x_{1}(t)-a_{22} x_{2}(t)\right) d t+\sigma_{2} d B_{2}(t)\right],
\end{aligned}
$$

where $B_{i}(t), i=1,2$ are mutually independent one dimensional standard Brownian motions with $B_{i}(0)=0, i=1,2$, and $\sigma_{i}, i=1,2$ are the intensities of white noise. It is shown in [8] that if $a_{11} a_{22}>a_{12} a_{21}$ then there is a unique nonnegative solution of system (2). For small white noise there is a stationary distribution of (2) and it has ergodic property. 
Biologically, this implies that with small perturbation of environment, the stability of the two species varies with the intensity of white noise, and both species will survive.

However, almost all known stochastic models assume that the growth rate and the carrying capacity of the population are independent of time $t$. In contrast, the natural growth rates of many populations vary with $t$ in real situation, for example, due to the seasonality. As a matter of fact, nonautonomous stochastic population systems have recently been studied by many authors, for example, [13-17].

In this paper we consider the system

$$
\begin{aligned}
d x_{1}(t)=x_{1}(t) & {\left[\left(r_{1}(t)-a_{11}(t) x_{1}(t)+a_{12}(t) x_{2}(t)\right) d t\right.} \\
& \left.+\sigma_{1}(t) d B_{1}(t)\right] \\
d x_{2}(t)=x_{2}(t)[ & \left(r_{2}(t)+a_{21}(t) x_{1}(t)-a_{22}(t) x_{2}(t)\right) d t \\
+ & \left.\sigma_{2}(t) d B_{2}(t)\right],
\end{aligned}
$$

where $r_{i}(t), a_{i j}(t), \sigma_{i}(t), i, j=1,2$ are all continuous bounded nonnegative functions on $[0,+\infty)$. The objective of our study is to investigate the long-time behavior of system (3). As in [8], we mainly discuss when the system is persistent and when it is not under a fewer conditions. More specifically, we show that there is a positive solution of system (3) and its pth moment bounded in Section 2. In Section 3, we deduce the persistence of the system. If the white noise is not large such that $r_{i}^{l}-\left(\left(\sigma_{i}^{u}\right)^{2} / 2\right)>0, i=1,2$, we will prove that the solution of system (3) is a stochastic persistence. In addition, we show that every component of the solution is persistent in mean. We further deduce that every component of the solution of system (3) is an asymptotic boundedness in mean. In Section 4, we show that larger white noise will make system (3) nonpersistent. Finally, we study the global attractivity of system (3).

Throughout this paper, unless otherwise specified, let $\left(\Omega,\left\{\mathscr{F}_{t}\right\}_{t \geq 0}, P\right)$ be a complete probability space with a filtration $\left\{\mathscr{F}_{t}\right\}_{t \geq 0}$ satisfying the usual conditions (i.e., it is right continuous and $\mathscr{F}_{0}$ contains all $P$-null sets). Let $R_{+}^{2}$ be the positive cone of $R^{2}$, namely, $R_{+}^{2}=\left\{x \in R^{2}: x_{i}>0, i=1,2\right\}$. If $x \in R^{n}$, its norm is denoted by $|x|=\left(\sum_{i=1}^{n} x_{i}^{2}\right)^{1 / 2}$. If $f(t)$ is a continuous bounded function on $[0,+\infty)$, we use the notation sup

$$
f^{u}=\sup _{t \in[0,+\infty)} f(t), \quad f^{l}=\min _{t \in[0,+\infty)} f(t) .
$$

\section{Existence and Uniqueness of the Positive Solution}

In population dynamics, the first concern is that the solution should be nonnegative. In order to do that a stochastic differential equation can have a unique global (i.e., no explosion at any finite time) solution for any given initial value, the coefficients of the equation are generally required to satisfy the linear growth condition and local Lipschitz condition (Mao [18]). However, the coefficients of system (3) do not satisfy the linear growth condition, though they are locally Lipschitz continuous, so the solution of system (3) may explode at a finite time. Following the way developed by Mao et al. [19], we show that there is a unique positive solution of (3).

Theorem 1. Assume that $a_{11}^{l} a_{22}^{l}>a_{12}^{u} a_{21}^{u}$. Then, there is a unique positive solution $x(t)=\left(x_{1}(t), x_{2}(t)\right)$ of system (3) on $t \geq 0$ for any given initial value $x(0) \in R_{+}^{2}$, and the solution will remain in $R_{+}^{2}$ with probability 1 , namely, $x(t) \in R_{+}^{2}$ for all $t \geq 0$ almost surely.

The proof of Theorem 1 is similar to [8]. But it is skilled in taking the value of $\epsilon$. We show it here.

Proof. Since the coefficients of the equation are locally Lipschitz continuous, for any given initial value $x(0) \in R_{+}^{2}$ there is an unique local solution $x(t)=\left(x_{1}(t), x_{2}(t)\right)$ on $t \in\left[0, \tau_{e}\right)$, where $\tau_{e}$ is the explosion time. To show that this solution is global, we need to show that $\tau_{e}=\infty$ a.s. Let $m_{0}>1$ be sufficiently large for every component of $x(0)$ lying within the interval $\left[1 / m_{0}, m_{0}\right]$. For each integer $m \geq m_{0}$, define the stopping time

$$
\begin{aligned}
\tau_{m}=\inf \{t & \in\left[0, \tau_{e}\right): \min \left\{x_{1}(t), x_{2}(t)\right\} \\
\leq & \left.\frac{1}{m} \text { or } \max \left\{x_{1}(t), x_{2}(t)\right\} \geq m\right\},
\end{aligned}
$$

where throughout this paper we set inf $\emptyset=\infty$ (as usual $\emptyset$ denotes the empty set). Clearly, $\tau_{m}$ is increasing as $m \rightarrow \infty$. Set $\tau_{\infty}=\lim _{m \rightarrow \infty} \tau_{m}$, whence $\tau_{\infty} \leq \tau_{e}$ a.s. If we can show that $\tau_{\infty}=\infty$ a.s., then $\tau_{e}=\infty$ a.s. and $x(t) \in R_{+}^{2}$ a.s. for all $t \geq 0$. In other words, to complete the proof, all we need to show is that $\tau_{\infty}=\infty$ a.s. If this statement is false, there is a pair of constant $T>0$ and $\varepsilon \in(0,1)$ such that

$$
P\left\{\tau_{\infty} \leq T\right\}>\varepsilon
$$

Hence, there is an integer $m_{1} \geq m_{0}$ such that

$$
P\left\{\tau_{m} \leq T\right\} \geq \varepsilon \quad \forall m \geq m_{1} .
$$

We define

$$
V(x)=a_{21}^{u}\left(x_{1}-1-\log x_{1}\right)+a_{12}^{u}\left(x_{2}-1-\log x_{2}\right) .
$$

By Itô's formula, we have

$$
\begin{aligned}
d V(x)=\{ & a_{21}^{u}\left(1-\frac{1}{x_{1}}\right) x_{1}\left[r_{1}(t)-a_{11}(t) x_{1}+a_{12}(t) x_{2}\right] \\
& +a_{12}^{u}\left(1-\frac{1}{x_{2}}\right) x_{2}\left[r_{2}(t)+a_{21}(t) x_{1}-a_{22}(t) x_{2}\right] \\
& \left.+\frac{1}{2}\left[a_{21}^{u} \sigma_{1}^{2}(t)+a_{12}^{u} \sigma_{2}^{2}(t)\right]\right\} d t
\end{aligned}
$$




$$
\begin{aligned}
& +a_{21}^{u} \sigma_{1}(t)\left(x_{1}-1\right) d B_{1}(t) \\
& +a_{12}^{u} \sigma_{2}(t)\left(x_{2}-1\right) d B_{2}(t) \\
:= & L V d t+a_{21}^{u} \sigma_{1}(t)\left(x_{1}-1\right) d B_{1}(t) \\
& +a_{12}^{u} \sigma_{2}(t)\left(x_{2}-1\right) d B_{2}(t),
\end{aligned}
$$

where

$$
\begin{aligned}
L V= & a_{21}^{u}\left(1-\frac{1}{x_{1}}\right) x_{1}\left[r_{1}(t)-a_{11}(t) x_{1}+a_{12}(t) x_{2}\right] \\
& +a_{12}^{u}\left(1-\frac{1}{x_{2}}\right) x_{2}\left[r_{2}(t)+a_{21}(t) x_{1}-a_{22}(t) x_{2}\right] \\
& +\frac{1}{2}\left[a_{21}^{u} \sigma_{1}^{2}(t)+a_{12}^{u} \sigma_{2}^{2}(t)\right] \\
\leq & a_{21}^{u}\left[\left(r_{1}^{u}+a_{11}^{u}\right) x_{1}-a_{12}^{l} x_{2}-a_{11}^{l} x_{1}^{2}\right. \\
& \left.+a_{12}^{u} x_{1} x_{2}-r_{1}^{l}+\frac{1}{2}\left(\sigma_{1}^{u}\right)^{2}\right] \\
+ & a_{12}^{u}\left[\left(r_{2}^{u}+a_{22}^{u}\right) x_{2}-a_{21}^{l} x_{1}-a_{22}^{l} x_{2}^{2}\right. \\
& \left.+a_{21}^{u} x_{1} x_{2}-r_{2}^{l}+\frac{1}{2}\left(\sigma_{2}^{u}\right)^{2}\right] .
\end{aligned}
$$

According to Young inequality, note that $x_{1} x_{2} \leq \epsilon x_{1}^{2}+$ $(1 / 4 \epsilon) x_{2}^{2}$, where $a_{21}^{u} / 2 a_{22}^{l}<\epsilon<a_{11}^{l} / 2 a_{12}^{u}$, then,

$$
\begin{aligned}
L V \leq & a_{21}^{u}\left[\left(r_{1}^{u}+a_{11}^{u}\right) x_{1}-a_{12}^{l} x_{2}-a_{11}^{l} x_{1}^{2}\right. \\
& \left.+a_{12}^{u}\left(\epsilon x_{1}^{2}+\frac{1}{4 \epsilon} x_{2}^{2}\right)-r_{1}^{l}+\frac{1}{2}\left(\sigma_{1}^{u}\right)^{2}\right] \\
+ & a_{12}^{u}\left[\left(r_{2}^{u}+a_{22}^{u}\right) x_{2}-a_{21}^{l} x_{1}-a_{22}^{l} x_{2}^{2}\right. \\
& \left.+a_{21}^{u}\left(\epsilon x_{1}^{2}+\frac{1}{4 \epsilon} x_{2}^{2}\right)-r_{2}^{l}+\frac{1}{2}\left(\sigma_{2}^{u}\right)^{2}\right] \\
= & -\left(a_{21}^{u} a_{11}^{l}-2 \epsilon a_{21}^{u} a_{12}^{u}\right) x_{1}^{2} \\
+ & {\left[a_{21}^{u}\left(r_{1}^{u}+a_{11}^{u}\right)-a_{12}^{u} a_{21}^{l}\right] x_{1} } \\
- & a_{21}^{u} r_{1}^{l}+\frac{1}{2} a_{21}^{u}\left(\sigma_{1}^{u}\right)^{2} \\
- & \left(a_{12}^{u} a_{22}^{l}-\frac{1}{2 \epsilon} a_{21}^{u} a_{12}^{u}\right) x_{2}^{2}
\end{aligned}
$$

$$
\begin{aligned}
& +\left[a_{12}^{u}\left(r_{2}^{u}+a_{22}^{u}\right)-a_{21}^{u} a_{12}^{l}\right] x_{2} \\
& -a_{12}^{u} r_{2}^{l}+\frac{1}{2} a_{12}^{u}\left(\sigma_{2}^{u}\right)^{2}
\end{aligned}
$$

$\leq K$.

Since $a_{21}^{u} / 2 a_{22}^{l}<\epsilon<a_{11}^{l} / 2 a_{12}^{u}$, we obtain $-\left(a_{21}^{u} a_{11}^{l}-2 \epsilon a_{12}^{u}\right)<$ 0 and $-\left(a_{12}^{u} a_{22}^{l}-(1 / 2 \epsilon) a_{21}^{u} a_{12}^{u}\right)<0$. Hence, $K$ is a positive constant. Integrating both sides of (9) from 0 to $\tau_{m} \wedge T$, we therefore obtain

$$
\begin{aligned}
& V\left(x\left(\tau_{m} \wedge T\right)\right)-V(x(0)) \\
& \quad \leq \int_{0}^{\tau_{m} \wedge T} K d t+\int_{0}^{\tau_{m} \wedge T} a_{21}^{u} \sigma_{1}(t)\left(x_{1}(t)-1\right) d B_{1}(t) \\
& \quad+\int_{0}^{\tau_{m} \wedge T} a_{12}^{u} \sigma_{2}(t)\left(x_{2}(t)-1\right) d B_{2}(t) .
\end{aligned}
$$

Whence, taking expectations yields

$$
\begin{aligned}
E\left[V\left(x\left(\tau_{m} \wedge T\right)\right)\right] & \leq V(x(0))+K E\left(\tau_{m} \wedge T\right) \\
& \leq V(x(0))+K T .
\end{aligned}
$$

Set $\Omega_{m}=\left\{\tau_{m} \leq T\right\}$ for $m \geq m_{1}$ and by (7), $P\left(\Omega_{m}\right) \geq \varepsilon$. Note that for every $\omega \in \Omega_{m}$, there is $x_{1}\left(\tau_{m}, \omega\right)$ or $x_{2}\left(\tau_{m}, \omega\right)$ equals either $m$ or $1 / m$, and therefore

$$
\begin{aligned}
& V\left(x\left(\tau_{m}, \omega\right)\right) \\
& \quad \geq \min \left\{a_{21}^{u}, a_{12}^{u}\right\}(m-1-\log m) \wedge\left(\frac{1}{m}-1-\log \frac{1}{m}\right) \\
& \quad:=h(m),
\end{aligned}
$$

where $\lim _{m \rightarrow \infty} h(m)=\infty$. It then follows from (13) that

$$
E[V(x(0))]+K T \geq E\left[1_{\Omega_{m}} \cdot V\left(x\left(\tau_{m}, \omega\right)\right)\right] \geq \epsilon h(m),
$$

where $1_{\Omega_{m}}$ is the indicator function of $\Omega_{m}$. Letting $m \rightarrow \infty$ leads to the contradiction

$$
\infty>V(x(0))+K T=\infty,
$$

so we must have $\tau_{\infty}=\infty$ a.s. This completes the proof of Theorem 1 .

Remark 2. By Theorem 1, we observe that for any given initial value $x(0) \in R_{+}^{2}$, there is a unique solution $x(t)=$ $\left(x_{1}(t), x_{2}(t)\right)$ of system (3) on $t \geq 0$ and the solution will remain in $R_{+}^{2}$ with probability 1 , no matter how large the intensities of white noise are. So, under the same assumption there is an global unique positive solution of the corresponding deterministic system of system (3).

Next, we show that the pth moment of the solution of system (3) is bounded in time average. 
Theorem 3. Assume that $a_{11}^{l} a_{22}^{l}>a_{12}^{u} a_{21}^{u}$. Then there exists a positive constant $K(p)$ such that the solution $x(t)$ of system (3) has the following property:

$$
E\left[c_{1} x_{1}^{p}(t)+c_{2} x_{2}^{p}(t)\right] \leq K(p), \quad \forall t \in[0, \infty), p>1,
$$

where $c_{1}, c_{2}$ satisfy

$$
\frac{\left(a_{21}^{u}\right)^{p+1}}{a_{11}^{l}\left(a_{22}^{l}\right)^{p}}<\frac{c_{1}}{c_{2}}<\frac{a_{22}^{l}\left(a_{11}^{l}\right)^{p}}{\left(a_{12}^{u}\right)^{p+1}}
$$

Proof. By Itô's formula, we have

$$
\begin{aligned}
d x_{1}^{p}(t)= & p x_{1}^{p}(t)\left[\left(r_{1}(t)-a_{11}(t) x_{1}(t)+a_{12}(t) x_{2}(t)\right) d t\right. \\
& \left.+\sigma_{1}(t) d B_{1}(t)\right] \\
+ & \frac{1}{2} p(p-1) x_{1}^{p}(t) \sigma_{1}^{2}(t) d t \\
= & p\left[\left(r_{1}(t)+\frac{p-1}{2} \sigma_{1}^{2}(t)\right) x_{1}^{p}(t)-a_{11}(t) x_{1}^{p+1}(t)\right. \\
& \left.+a_{12}(t) x_{1}^{p}(t) x_{2}(t)\right] d t \\
& +\sigma_{1}(t) p x_{1}^{p}(t) d B_{1}(t) \\
= & p\left[\alpha_{1}(t) x_{1}^{p}(t)-a_{11}(t) x_{1}^{p+1}(t)\right. \\
& \left.+a_{12}(t) x_{1}^{p}(t) x_{2}(t)\right] d t \\
& +\sigma_{1}(t) p x_{1}^{p}(t) d B_{1}(t) \\
\leq & p\left[\alpha_{1}^{u} x_{1}^{p}(t)-a_{11}^{l} x_{1}^{p+1}(t)+a_{12}^{u} x_{1}^{p}(t) x_{2}(t)\right] d t \\
& +p \sigma_{1}^{u} x_{1}^{p}(t) d B_{1}(t),
\end{aligned}
$$

where $\alpha_{1}(t)=r_{1}(t)+((p-1) / 2) \sigma_{1}^{2}(t)$, and

$$
\begin{aligned}
d x_{2}^{p}(t)= & p x_{2}^{p}(t)\left[\left(r_{2}(t)-a_{22}(t) x_{2}(t)+a_{21}(t) x_{1}(t)\right) d t\right. \\
& \left.+\sigma_{2}(t) d B_{2}(t)\right] \\
+ & \frac{1}{2} p(p-1) x_{2}^{p}(t) \sigma_{2}^{2}(t) d t \\
= & p\left[\left(r_{2}(t)+\frac{p-1}{2} \sigma_{2}^{2}(t)\right) x_{2}^{p}(t)-a_{22}(t) x_{2}^{p+1}(t)\right. \\
& \left.+a_{21}(t) x_{2}^{p}(t) x_{1}(t)\right] d t \\
+ & \sigma_{2}(t) p x_{2}^{p}(t) d B_{2}(t) \\
= & p\left[\alpha_{2}(t) x_{2}^{p}(t)-a_{22}(t) x_{2}^{p+1}(t)\right. \\
& \left.+a_{21}(t) x_{2}^{p}(t) x_{1}(t)\right] d t+\sigma_{2}(t) p x_{2}^{p}(t) d B_{2}(t) \\
\leq & p\left[\alpha_{2}^{u} x_{2}^{p}(t)-a_{22}^{l} x_{2}^{p+1}(t)+a_{21}^{u} x_{2}^{p}(t) x_{1}(t)\right] d t \\
+ & p \sigma_{2}^{u} x_{2}^{p}(t) d B_{2}(t),
\end{aligned}
$$

where $\alpha_{2}(t)=r_{2}(t)+((p-1) / 2) \sigma_{2}^{2}(t)$. According to Young inequality, we obtain

$$
x_{1}^{p}(t) x_{2}(t) \leq \epsilon_{1} x_{1}^{p+1}(t)+\frac{1}{p+1}\left(\frac{p}{p+1}\right)^{p}\left(\frac{1}{\epsilon_{1}}\right)^{p} x_{2}^{p+1}(t),
$$

$$
\epsilon_{1}=\frac{p a_{11}^{l}}{(p+1) a_{12}^{u}}
$$

$$
\begin{array}{r}
x_{2}^{p}(t) x_{1}(t) \leq \epsilon_{2} x_{2}^{p+1}(t)+\frac{1}{p+1}\left(\frac{p}{p+1}\right)^{p}\left(\frac{1}{\epsilon_{2}}\right)^{p} x_{1}^{p+1}(t), \\
\epsilon_{2}=\frac{p a_{22}^{l}}{(p+1) a_{21}^{u}} .
\end{array}
$$

Thus, we have

$$
\begin{aligned}
d x_{1}^{p}(t) \leq p\left[\alpha_{1}^{u} x_{1}^{p}(t)-a_{11}^{l} x_{1}^{p+1}(t)+a_{12}^{u} \epsilon_{1} x_{1}^{p+1}(t)\right. \\
\left.+a_{12}^{u} \frac{1}{p+1}\left(\frac{p}{p+1}\right)^{p}\left(\frac{1}{\epsilon_{1}}\right)^{p} x_{2}^{p+1}(t)\right] d t \\
+p \sigma_{1}^{u} x_{1}^{p}(t) d B_{1}(t), \\
d x_{2}^{p}(t) \leq p\left[\alpha_{2}^{u} x_{2}^{p}(t)-a_{22}^{l} x_{2}^{p+1}(t)+a_{21}^{u} \epsilon_{2} x_{2}^{p+1}(t)\right. \\
\left.+a_{21}^{u} \frac{1}{p+1}\left(\frac{p}{p+1}\right)^{p}\left(\frac{1}{\epsilon_{2}}\right)^{p} x_{1}^{p+1}(t)\right] d t \\
+p \sigma_{2}^{u} x_{2}^{p}(t) d B_{2}(t) .
\end{aligned}
$$

Since $a_{11}^{l} a_{22}^{l}>a_{12}^{u} a_{21}^{u}$, there exist two positive constants $c_{1}, c_{2}$ which satisfy

$$
\frac{\left(a_{21}^{u}\right)^{p+1}}{a_{11}^{l}\left(a_{22}^{l}\right)^{p}}<\frac{c_{1}}{c_{2}}<\frac{a_{22}^{l}\left(a_{11}^{l}\right)^{p}}{\left(a_{12}^{u}\right)^{p+1}}
$$

Therefore,

$$
\begin{aligned}
& d\left(c_{1} x_{1}^{p}(t)+c_{2} x_{2}^{p}(t)\right) \\
& \leq-p\left[\left(c_{1} a_{11}^{l}-c_{1} a_{12}^{u} \epsilon_{1}-c_{2} a_{21}^{u} \frac{p^{p}}{(p+1)^{p+1} \epsilon_{2}^{p}}\right) x_{1}^{p+1}(t)\right. \\
& +\left(c_{2} a_{22}^{l}-c_{1} a_{21}^{u} \epsilon_{2}-c_{1} a_{12}^{u} \frac{p^{p}}{(p+1)^{p+1} \epsilon_{1}^{p}}\right) x_{2}^{p+1}(t) \\
& \left.-\sum_{i=1}^{2} c_{i} \alpha_{i}^{u} x_{i}^{p}(t)\right] d t+\sum_{i=1}^{2} c_{i} p \sigma_{i}^{u} x_{i}^{p}(t) d B_{i}(t) .
\end{aligned}
$$

From (23) and the values of $\epsilon_{1}, \epsilon_{2}$, we obtain

$$
\frac{a_{21}^{u}\left(p^{p} /(p+1)^{p+1} \epsilon_{2}^{p}\right)}{a_{11}^{l}-a_{12}^{u} \epsilon_{1}}<\frac{c_{1}}{c_{2}}<\frac{a_{22}^{l}-a_{21}^{u} \epsilon_{2}}{a_{12}^{u}\left(p^{p} /(p+1)^{p+1} \epsilon_{1}^{p}\right)},
$$


which implies that $c_{1} a_{11}^{l}-c_{1} a_{12}^{u} \epsilon_{1}-c_{2} a_{21}^{u}\left(p^{p} /(p+1)^{p+1} \epsilon_{2}^{p}\right)>0$ and $c_{2} a_{22}^{l}-c_{1} a_{21}^{u} \epsilon_{2}-c_{1} a_{12}^{u}\left(p^{p} /\left((p+1)^{p+1} \epsilon_{1}^{p}\right)\right)>0$. Let

$\alpha=\max \left\{\alpha_{1}^{u}, \alpha_{2}^{u}\right\}$,

$$
\begin{array}{r}
\beta=\min \left\{c_{1}^{-(p+1) / p}\left[c_{1} a_{11}^{l}-c_{1} a_{12}^{u} \epsilon_{1}-c_{2} a_{21}^{u} \frac{p^{p}}{(p+1)^{p+1} \epsilon_{2}^{p}}\right],\right. \\
\left.c_{2}^{-(p+1) / p}\left[c_{2} a_{22}^{l}-c_{1} a_{21}^{u} \epsilon_{2}-c_{1} a_{12}^{u} \frac{p^{p}}{(p+1)^{p+1} \epsilon_{1}^{p}}\right]\right\},
\end{array}
$$

then we have

$$
\begin{aligned}
d\left(c_{1} x_{1}^{p}(t)+c_{2} x_{2}^{p}(t)\right) & \\
\leq p & {\left[\alpha\left(\sum_{i=1}^{2} c_{i} x_{i}^{p}(t)\right)-\beta\left(\sum_{i=1}^{2} c_{i}^{1+(1 / p)} x_{i}^{p+1}\right)\right] d t } \\
+ & \sum_{i=1}^{2} c_{i} p \sigma_{i}^{u} x_{i}^{p}(t) d B_{i}(t) .
\end{aligned}
$$

Hence, we get

$$
\begin{aligned}
& \frac{d E\left[c_{1} x_{1}^{p}(t)+c_{2} x_{2}^{p}(t)\right]}{d t} \\
& \leq p \alpha E\left[c_{1} x_{1}^{p}(t)+c_{2} x_{2}^{p}(t)\right] \\
& \quad-p \beta E\left[c_{1}^{1+(1 / p)} x_{1}^{p+1}(t)+c_{2}^{1+(1 / p)} x_{2}^{p+1}(t)\right] \\
& \leq p \alpha E\left[c_{1} x_{1}^{p}(t)+c_{2} x_{2}^{p}(t)\right] \\
& \quad-p \beta\left\{\left[E\left(c_{1} x_{1}^{p}(t)\right)\right]^{1+(1 / p)}\right. \\
& \left.\quad+\left[E\left(c_{2} x_{2}^{p}(t)\right)\right]^{1+(1 / p)}\right\} \\
& \leq p \alpha E\left[c_{1} x_{1}^{p}(t)+c_{2} x_{2}^{p}(t)\right] \\
& -p \beta \cdot 2^{-1 / p}\left[E\left(c_{1} x_{1}^{p}(t)+c_{2} x_{2}^{p}(t)\right)\right]^{1+(1 / p)} .
\end{aligned}
$$

By the comparison theorem, we get

$$
\limsup _{t \rightarrow \infty} E\left[c_{1} x_{1}^{p}(t)+c_{2} x_{2}^{p}(t)\right] \leq 2\left(\frac{\alpha}{\beta}\right)^{p}:=M(p),
$$

which implies that there is a $T_{0}>0$, such that

$$
E\left[c_{1} x_{1}^{p}(t)+c_{2} x_{2}^{p}(t)\right] \leq 2 M(p), \quad \forall t>T_{0} .
$$

Besides, note that $E\left[c_{1} x_{1}^{p}(t)+c_{2} x_{2}^{p}(t)\right]$ is continuous, then there is a $\widetilde{M}(p)>0$ such that

$$
E\left[c_{1} x_{1}^{p}(t)+c_{2} x_{2}^{p}(t)\right] \leq \widetilde{M}(p), \quad \forall t \in\left[0, T_{0}\right] .
$$

Let $K(p)=\max \{2 M(p), \widetilde{M}(p)\}$, then

$$
E\left[c_{1} x_{1}^{p}(t)+c_{2} x_{2}^{p}(t)\right] \leq K(p), \quad \forall t \in[0, \infty) .
$$

\section{Persistence}

Theorem 1 shows that the solution of system (3) will remain in the positive cone $R_{+}^{2}$ if $a_{11}^{l} a_{22}^{l}>a_{12}^{u} a_{21}^{u}$. Studying a population system, we pay more attention on whether the system is persistent. In this section, we first show that the solution is a stochastic permanence. Next we show that the solution is persistent in time average. Moreover, we show that the solution $x(t)$ of system (3) is an asymptotic boundedness in time average.

3.1. Stochastic Permanence. Let $y(t)$ be the solution of a randomized nonautonomous competitive equation:

$$
\begin{array}{r}
d y_{i}(t)=y_{i}(t)\left[\left(b_{i}(t)-\sum_{j=1}^{n} a_{i j}(t) y_{j}(t)\right) d t+\sigma_{i}(t) d B_{i}(t)\right], \\
i=1,2, \ldots, n
\end{array}
$$

where $B_{i}(t), i=1,2, \ldots, n$, are independent standard Brownian motions, $y(0)=y_{0}>0$ while $y_{0}$ is independent of $B(t)$, and $b_{i}(t), a_{i j}(t), \sigma_{i}(t)$ are all continuous bounded nonnegative functions on $[0,+\infty)$.

Lemma 4 (see [15]). Assume that $b_{i}^{l}-\left(\left(\sigma_{i}^{u}\right)^{2} / 2\right)>0, i=$ $1,2, \ldots, n$, then for any given initial value $y(0) \in R_{+}^{n}$, the solution $y(t)$ of (36) has the properties

$$
\limsup _{t \rightarrow \infty} E\left(\frac{1}{|y(t)|^{\theta}}\right) \leq H,
$$

where $H$ is a constant, $\theta$ is an arbitrary positive constant satisfying

$$
\theta \max _{1 \leq i \leq n}\left(\sigma_{i}^{u}\right)^{2}<2 \min _{1 \leq i \leq n}\left(b_{i}(t)-\frac{\sigma_{i}^{2}(t)}{2}\right)^{l} .
$$

Let $N(t)$ be the solution of a randomized nonautonomous logistic equation

$$
d N(t)=N(t)[(a(t)-b(t) N(t)) d t+\alpha(t) d B(t)],
$$

where $B(t)$ is a 1-dimensional standard Brownian motion, $N(0)=N_{0}>0$, and $N_{0}$ is independent of $B(t)$.

Lemma 5 (see [13]). Assume that $a(t), b(t)$, and $\alpha(t)$ are bounded continuous functions defined on $[0, \infty), a(t)>0$ and $b(t)>0$. Then there exists a unique continuous positive 
solution of (36) for any initial value $N(0)=N_{0}>0$, which is global and represented by

$$
\begin{array}{r}
N(t)=\exp \left\{\int_{0}^{t}\left[a(s)-\left(\frac{\alpha^{2}(s)}{2}\right)\right] d s+\alpha(s) d B(s)\right\} \\
\times\left(\left(\frac{1}{N_{0}}\right)+\int_{0}^{t} b(s) \exp \left\{\int_{0}^{s}\left[a(\tau)-\left(\frac{\alpha^{2}(\tau)}{2}\right)\right] d \tau\right.\right. \\
+\alpha(\tau) d B(\tau)\} d s)^{-1}, \\
t \geq 0 .
\end{array}
$$

From Lemma 4 we have the following.

Lemma 6. Assume that $a^{l}-\left(\left(\alpha^{u}\right)^{2} / 2\right)>0$, then for any given initial value $N(0) \in R_{+}$, the solution $N(t)$ of (36) has the properties

$$
\limsup _{t \rightarrow \infty} E\left(\frac{1}{N^{\theta}(t)}\right) \leq H
$$

where $H$ is a constant, $\theta$ is positive constant satisfying

$$
\theta\left(\alpha^{u}\right)^{2}<2\left[a^{l}-\frac{\left(\alpha^{u}\right)^{2}}{2}\right]
$$

Let $\phi(t)=\left(\phi_{1}(t), \phi_{2}(t)\right)^{T}$ be the solution of

$$
\begin{array}{r}
d \phi_{i}(t)=\phi_{i}(t)\left[\left(r_{i}(t)-a_{i i}(t) \phi_{i}(t)\right) d t+\sigma_{i}(t) d B_{i}(t)\right], \\
i=1,2,
\end{array}
$$

where $B_{i}(t), i=1,2$, are independent standard Brownian motions, $\phi(0)=\phi_{0}>0$, and $\phi_{0} \in R_{+}^{2}, r_{i}(t), a_{i i}(t), \sigma_{i}(t), i=$ 1,2 are all continuous bounded nonnegative functions on $[0,+\infty)$. From Lemma 4 it is easy to know the following.

Lemma 7. Assume that $\bar{r}_{i}^{l}=r_{i}^{l}-\left(\left(\sigma_{i}^{u}\right)^{2} / 2\right)>0, i=1,2$, then for any given initial value $\phi(0) \in R_{+}^{2}$, the solution $\phi(t)$ of (40) has the properties

$$
\limsup _{t \rightarrow \infty} E\left(\frac{1}{\phi_{i}^{\theta}(t)}\right) \leq H_{i}, \quad i=1,2,
$$

where $H_{i}, i=1,2$ are two constants, $\theta$ is positive constant satisfying

$$
\theta\left(\sigma_{i}^{u}\right)^{2}<2 \bar{r}_{i}^{l}, \quad i=1,2 .
$$

Lemma 8. Assume that $\bar{r}_{i}^{l}>0, i=1,2$, then for any given initial value $x_{0} \in R_{+}^{2}$, the solution $x(t)$ of system (3) has the properties

$$
\begin{gathered}
x_{i}(t) \geq \phi_{i}(t), \quad i=1,2, \\
\limsup _{t \rightarrow \infty} E\left(\frac{1}{x_{i}^{\theta}(t)}\right) \leq H_{i}, \quad i=1,2,
\end{gathered}
$$

where $H_{i}, i=1,2$ are two constants, $\theta$ is positive constant satisfying

$$
\theta\left(\sigma_{i}^{u}\right)^{2}<2 \bar{r}_{i}^{l}, \quad i=1,2
$$

Proof. Equation (43) follows directly from the classical comparison theorem of stochastic differential equations (see [20]). Thus, we obtain

$$
\limsup _{t \rightarrow \infty} E\left(\frac{1}{x_{i}^{\theta}(t)}\right) \leq \limsup _{t \rightarrow \infty} E\left(\frac{1}{\phi_{i}^{\theta}(t)}\right) \leq H_{i}, \quad i=1,2 .
$$

Definition 9. System (3) is said to be stochastically permanent if for any $\varepsilon \in(0,1)$, there exists a pair of positive constants $\delta=$ $\delta(\epsilon)$ and $M=M(\epsilon)$ such that for any initial value $x_{0} \in R_{+}^{2}$, the solution obeys

$$
\begin{gathered}
\liminf _{t \rightarrow \infty} P\left\{x_{i}(t) \leq M(\epsilon)\right\} \geq 1-\epsilon, \\
\liminf _{t \rightarrow \infty} P\left\{x_{i}(t) \geq \delta(\epsilon)\right\} \geq 1-\epsilon, \quad i=1,2 .
\end{gathered}
$$

Theorem 10. Assume that $a_{11}^{l} a_{22}^{l}>a_{12}^{u} a_{21}^{u}, \bar{r}_{i}^{l}>0, i=1,2$, then system (3) is stochastically permanent.

The proof is a simple application of the Chebyshev inequality, we omit it.

3.2. Persistence in Time Average. Theorem 10 shows that if the white noise is not large, the solution of system (3) is survive with large probability. In this part, we show $x(t)$ is persistence in mean.

Lemma 11. Assume that $\bar{r}_{i}^{l}>0, i=1,2$, then for any given initial value $\phi(0) \in R_{+}^{2}$, the solution $\phi(t)$ of (40) has the properties

$$
\begin{array}{r}
z_{i}(t) e^{-\left[\max _{0 \leq s \leq t} \int_{0}^{s} \sigma_{i}(\tau) d B_{i}(\tau)-\int_{0}^{t} \sigma_{i}(\tau) d B_{i}(\tau)\right]} \\
\leq \phi_{i}(t) \leq z_{i}(t) e^{-\left[\min _{0 \leq s \leq t} \int_{0}^{s} \sigma_{i}(\tau) d B_{i}(\tau)-\int_{0}^{t} \sigma_{i}(\tau) d B_{i}(\tau)\right]}, \\
i=1,2,
\end{array}
$$

where $z(t)=\left(z_{1}(t), z_{2}(t)\right)$ is the solution of

$$
\begin{array}{r}
d z_{i}(t)=z_{i}(t)\left[r_{i}(t)-\frac{\sigma_{i}^{2}(t)}{2}-a_{i i}^{u} z_{i}(t)\right] d t, \\
z_{i}(0)=\phi_{i}(0), i=1,2 .
\end{array}
$$


Proof. From Lemma 5, we know

$$
\begin{aligned}
& \frac{1}{\phi_{i}(t)}=\frac{1}{\phi_{i}(0)} e^{-\int_{0}^{t}\left[r_{i}(s)-\left(\sigma_{i}^{2}(s) / 2\right)\right] d s+\sigma_{i}(s) d B_{i}(s)} \\
& +a_{i i}^{u} \int_{0}^{t} e^{-\int_{0}^{t}\left[r_{i}(s)-\left(\sigma_{i}^{2}(s) / 2\right)\right] d s+\sigma_{i}(s) d B_{i}(s)} \\
& \times e^{+\int_{0}^{s}\left[r_{i}(\tau)-\left(\sigma_{i}^{2}(\tau) / 2\right)\right] d \tau+\sigma_{i}(\tau) d B_{i}(\tau)} d s \\
& =e^{-\int_{0}^{t} \sigma_{i}(s) d B_{i}(s)}\left[\frac{1}{\phi_{i}(0)} e^{-\int_{0}^{t}\left[r_{i}(s)-\left(\sigma_{i}^{2}(s) / 2\right)\right] d s}\right. \\
& +a_{i i}^{u} \int_{0}^{t} e^{-\int_{s}^{t}\left[r_{i}(\tau)-\left(\sigma_{i}^{2}(\tau) / 2\right)\right] d \tau} \\
& \left.\times e^{\int_{0}^{s} \sigma_{i}(\tau) d B_{i}(\tau)} d s\right] \\
& \leq e^{-\int_{0}^{t} \sigma_{i}(s) d B_{i}(s)}\left[\frac{1}{\phi_{i}(0)} e^{-\int_{0}^{t}\left[r_{i}(s)-\left(\sigma_{i}^{2}(s) / 2\right)\right] d s}\right. \\
& +a_{i i}^{u} e^{\max _{0 \leq s \leq t}\left(\int_{0}^{s} \sigma_{i}(\tau) d B_{i}(\tau)\right)} \\
& \left.\times \int_{0}^{t} e^{-\int_{s}^{t}\left[r_{i}(\tau)-\left(\sigma_{i}^{2}(\tau) / 2\right)\right] d \tau} d s\right] \\
& \leq \frac{e^{\max _{0 \leq s \leq t}\left[\int_{0}^{s} \sigma_{i}(\tau) d B_{i}(\tau)\right]-\int_{0}^{t} \sigma_{i}(s) d B_{i}(s)}}{z_{i}(t)} .
\end{aligned}
$$

Similarly, we have

$$
\begin{aligned}
& \frac{1}{\phi_{i}(t)} \geq e^{-\int_{0}^{t} \sigma_{i}(s) d B_{i}(s)}\left[\frac{1}{\phi_{i}(0)} e^{-\int_{0}^{t}\left[r_{i}(s)-\left(\sigma_{i}^{2}(s) / 2\right)\right] d s}\right. \\
& +a_{i i}^{u} e^{\min _{0 \leq s \leq t}\left(\int_{0}^{s} \sigma_{i}(\tau) d B_{i}(\tau)\right)} \\
& \left.\times \int_{0}^{t} e^{-\int_{s}^{t}\left[r_{i}(\tau)-\left(\sigma_{i}^{2}(\tau) / 2\right)\right] d \tau} d s\right] \\
& \geq \frac{e^{\min _{0 \leq s \leq t}\left[\int_{0}^{s} \sigma_{i}(\tau) d B_{i}(\tau)\right]-\int_{0}^{t} \sigma_{i}(s) d B_{i}(s)}}{z_{i}(t)} .
\end{aligned}
$$

Lemma 12. Assume that $\bar{r}_{i}^{l}>0, i=1,2$, then for any given initial value $z(0) \in R_{+}^{2}$, the solution $z(t)$ of (49) has the following properties

$$
\begin{gathered}
\widetilde{z}_{i}(t) \leq z_{i}(t) \leq \widehat{z}_{i}(t), \\
\lim _{t \rightarrow \infty} \widetilde{z}_{i}(t)=\frac{\bar{r}_{i}^{l}}{a_{i i}^{u}}, \quad \lim _{t \rightarrow \infty} \widehat{z}_{i}(t)=\frac{\bar{r}_{i}^{u}}{a_{i i}^{u}},
\end{gathered}
$$

where $\tilde{z}(t)=\left(\widetilde{z}_{1}(t), \widetilde{z}_{2}(t)\right), \widehat{z}(t)=\left(\widehat{z}_{1}(t), \widehat{z}_{2}(t)\right)$ are the solutions of the two equations, respectively,

$$
\begin{aligned}
& d \widetilde{z}_{i}(t)=\widetilde{z}_{i}(t)\left[\bar{r}_{i}^{l}-a_{i i}^{u} \widetilde{z}_{i}(t)\right] d t, \quad \widetilde{z}_{i}(0)=z_{i}(0), i=1,2, \\
& d \widehat{z}_{i}(t)=\widehat{z}_{i}(t)\left[\bar{r}_{i}^{u}-a_{i i}^{u} \widehat{z}_{i}(t)\right] d t, \quad \widehat{z}_{i}(0)=z_{i}(0), i=1,2 .
\end{aligned}
$$

Proof. Let $\widetilde{z}(t)=\left(\widetilde{z}_{1}(t), \widetilde{z}_{2}(t)\right), \widehat{z}(t)=\left(\widehat{z}_{1}(t), \widehat{z}_{2}(t)\right)$ are the solutions of SDE (53) and (54), respectively, with the positive initial value $z(0)$. By Lemma 5 , we know

$$
\begin{aligned}
& \widetilde{z}_{i}(t)=\frac{e^{\bar{r}_{i}^{l} t}}{1 / \widetilde{z}_{i}(0)+\left(a_{i i}^{u} / \bar{r}_{i}^{l}\right)\left(e^{\bar{r}_{i}^{l} t}-1\right)}, \\
& \widehat{z}_{i}(t)=\frac{e^{\bar{r}_{i}^{u} t}}{1 / \widehat{z}_{i}(0)+\left(a_{i i}^{u} / \bar{r}_{i}^{u}\right)\left(e^{\bar{r}_{i}^{u} t}-1\right)} .
\end{aligned}
$$

Thus,

$$
\lim _{t \rightarrow \infty} \widetilde{z}_{i}(t)=\frac{\bar{r}_{i}^{l}}{a_{i i}^{u}}, \quad \lim _{t \rightarrow \infty} \widehat{z}_{i}(t)=\frac{\bar{r}_{i}^{u}}{a_{i i}^{u}} .
$$

By the classical comparison theorem of ordinary differential equations, we know

$$
\widetilde{z}_{i}(t) \leq z_{i}(t) \leq \widehat{z}_{i}(t) .
$$

Lemma 13. Assume that $\bar{r}_{i}^{l}>0, i=1,2$, then for any given initial value $\phi(0) \in R_{+}^{2}$, the solution $\phi(t)$ of (40) has the properties

$$
\lim _{t \rightarrow \infty} \frac{\log \phi_{i}(t)}{t}=0, \quad \text { a.s. }
$$

Proof. By Lemma 12, we know

$$
\begin{aligned}
& e^{-\left[\max _{0 \leq s \leq t} \int_{0}^{s} \sigma_{i}(\tau) d B_{i}(\tau)-\int_{0}^{t} \sigma_{i}(\tau) d B_{i}(\tau)\right]} \\
& \quad \leq \frac{\phi_{i}(t)}{z_{i}(t)} \leq e^{-\left[\min _{0 \leq s \leq t} \int_{0}^{s} \sigma_{i}(\tau) d B_{i}(\tau)-\int_{0}^{t} \sigma_{i}(\tau) d B_{i}(\tau)\right]} .
\end{aligned}
$$

So, we have

$$
\begin{gathered}
\int_{0}^{t} \sigma_{i}(\tau) d B_{i}(\tau)-\max _{0 \leq s \leq t} \int_{0}^{s} \sigma_{i}(\tau) d B_{i}(\tau) \\
\leq \log \phi_{i}(t)-\log z_{i}(t) \\
\leq \int_{0}^{t} \sigma_{i}(\tau) d B_{i}(\tau) \\
\quad-\min _{0 \leq s \leq t} \int_{0}^{s} \sigma_{i}(\tau) d B_{i}(\tau)
\end{gathered}
$$

Let $M_{i}(t)=\int_{0}^{t} \sigma_{i}(\tau) d B_{i}(\tau)$, then

$$
\left\langle M_{i}, M_{i}\right\rangle_{t}=\int_{0}^{t} \sigma_{i}^{2}(\tau) d \tau .
$$


Since $\sigma_{i}(t), i=1,2$ are bounded, then

$$
\lim _{t \rightarrow \infty} \frac{\left\langle M_{i}, M_{i}\right\rangle_{t}}{t}=\lim _{t \rightarrow \infty} \frac{1}{t} \int_{0}^{t} \sigma_{i}^{2}(\tau) d \tau<\infty, \quad \text { a.s. }
$$

By the strong law of large numbers, we know

$$
\lim _{t \rightarrow \infty} \frac{M_{i}(t)}{t}=\lim _{t \rightarrow \infty} \frac{\int_{0}^{t} \sigma_{i}(\tau) d B_{i}(\tau)}{t}=0, \quad \text { a.s. }
$$

Thus,

$$
\lim _{t \rightarrow \infty} \max _{0 \leq s \leq t}\left|\frac{M_{i}(s)}{t}\right|=0, \quad \text { a.s. }
$$

Then from (60) we obtain

$$
\lim _{t \rightarrow \infty} \frac{\log \phi_{i}(t)}{t}=0, \quad \text { a.s. }
$$

Lemma 14. Assume that $\bar{r}_{i}^{l}>0, i=1,2$, then for any given initial value $\phi(0) \in R_{+}^{2}$, the solution $\phi(t)$ of (40) has the properties

$$
\liminf _{t \rightarrow \infty} \frac{1}{t} \int_{0}^{t} \phi_{i}(s) d s \geq \frac{\bar{r}_{i}^{l}}{a_{i i}^{u}}, \quad \text { a.s. }
$$

Proof. By Itô's formula, we have

$$
d \log \phi_{i}(t)=\left[r_{i}(t)-\frac{\sigma_{i}^{2}(t)}{2}-a_{i i}^{u} \phi_{i}(t)\right] d t+\sigma_{i}(t) d B_{i}(t) .
$$

Integrating both sides of this equation from 0 to $t$ yields

$$
\begin{aligned}
\frac{\log \phi_{i}(t)}{t}-\frac{\log \phi_{i}(0)}{t}= & \frac{\int_{0}^{t}\left[r_{i}(s)-\left(\sigma_{i}^{2}(s) / 2\right)\right] d s}{t} \\
& -\frac{a_{i i}^{u} \int_{0}^{t} \phi_{i}(s) d s}{t}+\frac{\int_{0}^{t} \sigma_{i}(s) d B_{i}(s)}{t} .
\end{aligned}
$$

By Lemma 13, we know that

$$
\lim _{t \rightarrow \infty} \frac{\int_{0}^{t} \sigma_{i}(s) d B_{i}(s)}{t}=\lim _{t \rightarrow \infty} \frac{\log \phi_{i}(t)}{t}=0, \quad \text { a.s. }
$$

Hence,

$$
\begin{aligned}
\liminf _{t \rightarrow \infty} \frac{1}{t} \int_{0}^{t} \phi_{i}(s) d s & =\frac{1}{a_{i i}^{u}} \liminf _{t \rightarrow \infty} \frac{1}{t} \int_{0}^{t}\left[r_{i}(s)-\frac{\sigma_{i}^{2}(s)}{2}\right] d s \\
& \geq \frac{\bar{r}_{i}^{l}}{a_{i i}^{u}}, \quad \text { a.s. }
\end{aligned}
$$

Definition 15. System (3) is said to be persistent in time average if

$$
\liminf _{t \rightarrow \infty} \frac{1}{t} \int_{0}^{t} x_{i}(s) d s>0, \quad i=1,2 .
$$

Theorem 16. Assume that $a_{11}^{l} a_{22}^{l}>a_{12}^{u} a_{21}^{u}$ and $\vec{r}_{i}^{l}>0, i=$ 1,2 , then the solution $x(t)$ of system (3) with any initial value $x(0) \in R_{+}^{2}$ has the following property:

$$
\begin{gathered}
\liminf _{t \rightarrow \infty} \frac{1}{t} \int_{0}^{t} x_{i}(s) d s \geq \frac{\bar{r}_{i}^{l}}{a_{i i}^{u}}, \\
\liminf _{t \rightarrow \infty} \frac{\log x_{i}(t)}{t} \geq 0, \quad \text { a.s., }
\end{gathered}
$$

and so system (3) is persistent in time average.

Proof. By Lemma 8, we know that

$$
x_{i}(t) \geq \phi_{i}(t) \quad i=1,2,
$$

where $\phi(t)=\left(\phi_{1}(t), \phi_{2}(t)\right)$ is the solution of system (40). Moreover, by Lemma 14 we know that

$$
\liminf _{t \rightarrow \infty} \frac{1}{t} \int_{0}^{t} x_{i}(s) d s \geq \liminf _{t \rightarrow \infty} \frac{1}{t} \int_{0}^{t} \phi_{i}(s) d s \geq \frac{\bar{r}_{i}^{l}}{a_{i i}^{u}}, \quad \text { a.s. }
$$

Hence, by Lemma 13 we know that

$$
\liminf _{t \rightarrow \infty} \frac{\log x_{i}(t)}{t} \geq \liminf _{t \rightarrow \infty} \frac{\log \phi_{i}(t)}{t}=0, \quad \text { a.s. }
$$

3.3. Asymptotic Boundedness of Integral Average. Theorem 16 shows that every component of the solution $x(t)$ of system (3) will survive forever in time average, if the white noise is not large. In this part, we further deduce that every component of $x(t)$ of system (3) will be an asymptotic boundedness in time average. Before we give the result, we do some preparation work.

Lemma 17. Let $f \in C[[0, \infty) \times \Omega,(0, \infty)], F(t) \in((0, \infty) \times$ $\Omega, R)$. If there exist positive constants $\lambda_{0}$ and $\lambda$ such that

$$
\log f(t) \geq \lambda t-\lambda_{0} \int_{0}^{t} f(s) d s+F(t), \quad t \geq 0, \text { a.s. }
$$

and $\lim _{t \rightarrow \infty}(F(t) / t)=0$ a.s., then

$$
\liminf _{t \rightarrow \infty} \frac{1}{t} \int_{0}^{t} f(s) d s \geq \frac{\lambda}{\lambda_{0}}, \text { a.s. }
$$

Proof. The proof is similar to the proof of Lemma in [21]. Let

$$
\varphi(t)=\int_{0}^{t} f(s) d s
$$

Since $f \in C[[0, \infty) \times \Omega,(0, \infty)], \varphi(t)$ is differentiable on $[0, \infty)$ and

$$
\frac{d \varphi(t)}{d t}=f(t)>0, \quad \text { for } t \geq 0
$$


Substituting $d \varphi(t) / d t$ and $\varphi(t)$ into (76), we obtain the following:

$$
\log \frac{d \varphi(t)}{d t} \geq \lambda t-\lambda_{0} \varphi(t)+F(t)
$$

thus

$$
e^{\lambda_{0} \varphi(t)} \frac{d \varphi(t)}{d t} \geq e^{\lambda t+F(t)}, \quad \text { for } t \geq 0
$$

Note that $\lim _{t \rightarrow \infty}(F(t) / t)=0$ a.s., then for $0<\varepsilon<$ $\min \{1, \lambda\}, \exists T=T(\omega)>0$ and $\Omega_{\varepsilon} \subset \Omega$ such that $P\left(\Omega_{\varepsilon}\right)>1-\varepsilon$ and $F(t) \geq-\varepsilon t, t \geq T, \omega \in \Omega_{\varepsilon}$. Then we have

$$
e^{\lambda_{0} \varphi(t)} \frac{d \varphi(t)}{d t} \geq e^{(\lambda-\varepsilon) t}, \quad \text { for } t \geq T, \omega \in \Omega_{\varepsilon} .
$$

Integrating inequality (82) from 0 to $t$ results in the following:

$$
\lambda_{0}^{-1}\left[e^{\lambda_{0} \varphi(t)}-e^{\lambda_{0} \varphi(T)}\right] \geq(\lambda-\varepsilon)^{-1}\left[e^{(\lambda-\varepsilon) t}-e^{(\lambda-\varepsilon) T}\right] .
$$

This inequality can be rewritten into

$$
e^{\lambda_{0} \varphi(t)} \geq e^{\lambda_{0} \varphi(T)}+\lambda_{0}(\lambda-\varepsilon)^{-1}\left[e^{(\lambda-\varepsilon) t}-e^{(\lambda-\varepsilon) T}\right] .
$$

Taking the logarithm of both sides and dividing both sides by $t(>0)$ yields

$$
\frac{\varphi(t)}{t} \geq \lambda_{0}^{-1} \frac{\log \left\{e^{\lambda_{0} \varphi(T)}+\lambda_{0}(\lambda-\varepsilon)^{-1}\left[e^{(\lambda-\varepsilon) t-e^{(\lambda-\varepsilon) T}}\right]\right\}}{t} .
$$

Then,

$$
\liminf _{t \rightarrow \infty} \frac{\varphi(t)}{t} \geq \frac{\lambda-\varepsilon}{\lambda_{0}}, \quad \omega \in \Omega_{\varepsilon} .
$$

Letting $\varepsilon \rightarrow \infty$ yields

$$
\liminf _{t \rightarrow \infty} \frac{1}{t} \int_{0}^{t} f(s) d s \geq \frac{\lambda}{\lambda_{0}}, \quad \text { a.s. }
$$

This finishes the proof of the Lemma.

Theorem 18. Assume that $a_{11}^{l} a_{22}^{l}>a_{12}^{u} a_{21}^{u}$ and $\bar{r}_{i}^{l}>0, i=$ 1,2 , then the solution $x(t)$ of system (3) with any initial value $x(0) \in R_{+}^{2}$ has the property

$$
\tilde{x}_{i}^{*} \leq \lim _{t \rightarrow \infty} \frac{1}{t} \int_{0}^{t} x_{i}(s) d s \leq \widehat{x}_{i}^{*}, \quad i=1,2, \text { a.s. }
$$

where

$$
\begin{array}{ll}
\tilde{x}_{1}^{*}=\frac{a_{22}^{u} \bar{r}_{1}^{l}+a_{12}^{l} \bar{r}_{2}^{l}}{a_{11}^{u} a_{22}^{u}-a_{12}^{l} a_{21}^{l}}, & \tilde{x}_{2}^{*}=\frac{a_{11}^{u} \bar{r}_{2}^{l}+a_{21}^{l} \bar{r}_{1}^{l}}{a_{11}^{u} a_{22}^{u}-a_{12}^{l} a_{21}^{l}}, \\
\widehat{x}_{1}^{*}=\frac{a_{22}^{l} \bar{r}_{1}^{u}+a_{12}^{u} \bar{r}_{2}^{u}}{a_{11}^{l} a_{22}^{l}-a_{12}^{u} a_{21}^{u}}, & \widehat{x}_{2}^{*}=\frac{a_{11}^{l} \bar{r}_{2}^{u}+a_{21}^{u} \bar{r}_{1}^{u}}{a_{11}^{l} a_{22}^{l}-a_{12}^{u} a_{21}^{u}} .
\end{array}
$$

Proof. To prove the results, we only need to prove

$$
\begin{aligned}
& \liminf _{t \rightarrow \infty} \frac{1}{t} \int_{0}^{t} x_{i}(s) d s \geq \widetilde{x}_{i}^{*}, \quad i=1,2, \text { a.s. } \\
& \limsup _{t \rightarrow \infty} \frac{1}{t} \int_{0}^{t} x_{i}(s) d s \leq \widehat{x}_{i}^{*}, \quad i=1,2 \text {, a.s. }
\end{aligned}
$$

By Itô's formula, we have

$$
\begin{aligned}
& d \log x_{1}(t) \\
& =\left[r_{1}(t)-\frac{1}{2} \sigma_{1}^{2}(t)-a_{11}(t) x_{1}(t)+a_{12}(t) x_{2}(t)\right] d t \\
& +\sigma_{1}(t) d B_{1}(t) \\
& =\left[r_{2}(t)-\frac{1}{2} \sigma_{2}^{2}(t)+a_{21}(t) x_{1}(t)-a_{22}(t) x_{2}(t)\right] d t \\
& +\sigma_{2}(t) d B_{2}(t) \text {. }
\end{aligned}
$$

First, we prove (91). Integrating both sides of (92) from 0 to $t$ yields

$$
\begin{aligned}
\log x_{1}(t)= & \log x_{1}(0)+\int_{0}^{t} \bar{r}_{1}(s) d s-\int_{0}^{t} a_{11}(s) x_{1}(s) d s \\
& +\int_{0}^{t} a_{12}(s) x_{2}(s) d s+\int_{0}^{t} \sigma_{1}(s) d B_{1}(s), \\
\log x_{2}(t)= & \log x_{2}(0)+\int_{0}^{t} \bar{r}_{2}(s) d s-\int_{0}^{t} a_{22}(s) x_{2}(s) d s \\
& +\int_{0}^{t} a_{21}(s) x_{1}(s) d s+\int_{0}^{t} \sigma_{2}(s) d B_{2}(s),
\end{aligned}
$$

where $\bar{r}_{i}(s)=r_{i}(s)-(1 / 2) \sigma_{i}^{2}(s), i=1,2$. Since $x_{i}(t)>0, i=$ 1,2 , hence

$$
\begin{aligned}
\log x_{1}(t) \leq & \log x_{1}(0)+\bar{r}_{1}^{u} t-a_{11}^{l} \int_{0}^{t} x_{1}(s) d s \\
& +a_{12}^{u} \int_{0}^{t} x_{2}(s) d s+\int_{0}^{t} \sigma_{1}(s) d B_{1}(s), \\
\log x_{2}(t) \leq & \log x_{2}(0)+\bar{r}_{2}^{u} t-a_{22}^{l} \int_{0}^{t} x_{2}(s) d s \\
& +a_{21}^{u} \int_{0}^{t} x_{1}(s) d s+\int_{0}^{t} \sigma_{2}(s) d B_{2}(s) .
\end{aligned}
$$


So we have

$$
\begin{aligned}
& a_{22}^{l} \log x_{1}(t)+a_{12}^{u} \log x_{2}(t) \\
& \leq a_{22}^{l}\left[\log x_{1}(0)+\int_{0}^{t} \sigma_{1}(s) d B_{1}(s)\right]+a_{22}^{l} \bar{r}_{1}^{u} t \\
& +a_{12}^{u}\left[\log x_{2}(0)+\int_{0}^{t} \sigma_{2}(s) d B_{2}(s)\right]+a_{12}^{u} \bar{r}_{2}^{u} t \\
& \quad-\left(a_{11}^{l} a_{22}^{l}-a_{21}^{u} a_{12}^{u}\right) \int_{0}^{t} x_{1}(s) d s .
\end{aligned}
$$

By Theorem 16, we know that

$$
\liminf _{t \rightarrow \infty} \frac{\log x_{i}(t)}{t} \geq 0, \quad i=1,2, \text { a.s. }
$$

Obviously,

$$
\lim _{t \rightarrow \infty} \frac{\log x_{i}(0)+\int_{0}^{t} \sigma_{i}(s) d B_{i}(s)}{t}=0, \quad i=1,2, \text { a.s. }
$$

Hence, we have

$$
\limsup _{t \rightarrow \infty} \frac{1}{t} \int_{0}^{t} x_{1}(s) d s \leq \frac{a_{22}^{l} \bar{r}_{1}^{u}+a_{12}^{u} \bar{r}_{2}^{u}}{a_{11}^{l} a_{22}^{l}-a_{21}^{u} a_{12}^{u}} \triangleq \widehat{x}_{1}^{*}, \quad \text { a.s. }
$$

Similarly, we have

$$
\limsup _{t \rightarrow \infty} \frac{1}{t} \int_{0}^{t} x_{2}(s) d s \leq \frac{a_{11}^{l} \bar{r}_{2}^{u}+a_{12}^{u} \bar{r}_{1}^{u}}{a_{11}^{l} a_{22}^{l}-a_{21}^{u} a_{12}^{u}} \triangleq \widehat{x}_{2}^{*}, \quad \text { a.s. }
$$

Next, we prove that (90) is true. Taking integration both sides of (92) from 0 to $t$, we have

$$
\begin{aligned}
\log x_{1}(t) \geq & \log x_{1}(0)+\bar{r}_{1}^{l} t-a_{11}^{u} \int_{0}^{t} x_{1}(s) d s \\
& +a_{12}^{l} \int_{0}^{t} x_{2}(s) d s+\int_{0}^{t} \sigma_{1}(s) d B_{1}(s), \\
\log x_{2}(t) \geq & \log x_{2}(0)+\bar{r}_{2}^{l} t-a_{22}^{u} \int_{0}^{t} x_{2}(s) d s \\
& +a_{21}^{l} \int_{0}^{t} x_{1}(s) d s+\int_{0}^{t} \sigma_{2}(s) d B_{2}(s) .
\end{aligned}
$$

By Theorem 16 we know that

$$
\begin{aligned}
& \liminf _{t \rightarrow \infty} \frac{1}{t} \int_{0}^{t} x_{1}(s) d s \geq \frac{\bar{r}_{1}^{l}}{a_{11}^{u}} \triangleq M_{1}, \quad \text { a.s., } \\
& \liminf _{t \rightarrow \infty} \frac{1}{t} \int_{0}^{t} x_{2}(s) d s \geq \frac{\bar{r}_{2}^{l}}{a_{22}^{u}} \triangleq N_{1}, \quad \text { a.s., }
\end{aligned}
$$

then for any $\varepsilon>0$, there is a $T(\omega)>0$ such that

$$
\frac{1}{t} \int_{0}^{t} x_{2}(s) d s \geq N_{1}-\varepsilon
$$

for $t>T(\omega)$. It follows from (100) that, for $t>T(\omega)$,

$$
\begin{aligned}
\log x_{1}(t) \geq & \log x_{1}(0)+\bar{r}_{1}^{l} t-a_{11}^{u} \int_{0}^{t} x_{1}(s) d s \\
& +a_{12}^{l}\left(N_{1}-\varepsilon\right) t+\int_{0}^{t} \sigma_{1}(s) d B_{1}(s) \\
= & \log x_{1}(0)-a_{11}^{u} \int_{0}^{t} x_{1}(s) d s \\
& +\left[\bar{r}_{1}^{l}+a_{12}^{l}\left(N_{1}-\varepsilon\right)\right] t+\int_{0}^{t} \sigma_{1}(s) d B_{1}(s) .
\end{aligned}
$$

From Lemma 17, we have

$$
\liminf _{t \rightarrow \infty} \frac{1}{t} \int_{0}^{t} x_{1}(s) d s \geq \frac{\bar{r}_{1}^{l}+a_{12}^{l}\left(N_{1}-\varepsilon\right)}{a_{11}^{u}}:=M_{2}>M_{1} \text {. }
$$

Similarly, we have

$$
\liminf _{t \rightarrow \infty} \frac{1}{t} \int_{0}^{t} x_{2}(s) d s \geq \frac{\bar{r}_{2}^{l}+a_{21}^{l}\left(M_{1}-\varepsilon\right)}{a_{22}^{u}}:=N_{2}>N_{1} .
$$

Continuing this process, we obtain two sequences $M_{n}$, $N_{n}(n=1,2, \ldots)$ such that

$$
\begin{aligned}
& M_{n}=\frac{\bar{r}_{1}^{l}+a_{12}^{l}\left(N_{n-1}-\varepsilon\right)}{a_{11}^{u}}, \\
& N_{n}=\frac{\bar{r}_{2}^{l}+a_{21}^{l}\left(M_{n-1}-\varepsilon\right)}{a_{22}^{u}} .
\end{aligned}
$$

By induction, we can easily show that $M_{n+1}>M_{n}, N_{n+1}>$ $N_{n}, n=1,2, \ldots$, that is, sequences $\left\{M_{n}, n=1,2, \ldots\right\}$ and $\left\{N_{n}, n=1,2, \ldots\right\}$ are nondecreasing. Moreover, note that (98) and (99), then the sequences $\left\{M_{n}, n=1,2, \ldots\right\}$ and $\left\{N_{n}, n=\right.$ $1,2, \ldots\}$, have upper bounds. Therefore, there are two positive $M, N$ such that

$$
\begin{aligned}
& \lim _{n \rightarrow \infty} M_{n}=M, \quad \lim _{n \rightarrow \infty} N_{n}=N, \\
& \liminf _{t \rightarrow \infty} \frac{1}{t} \int_{0}^{t} x_{1}(s) d s \geq M, \quad \liminf _{t \rightarrow \infty} \frac{1}{t} \int_{0}^{t} x_{2}(s) d s \geq N,
\end{aligned}
$$

which together with (106) implies

$$
\begin{aligned}
& a_{11}^{u} M-a_{12}^{l} N=\bar{r}_{1}^{l}-\varepsilon a_{12}^{l}, \\
& a_{22}^{u} N-a_{21}^{l} M=\bar{r}_{2}^{l}-\varepsilon a_{21}^{l} .
\end{aligned}
$$

Letting $\varepsilon \rightarrow 0$ yields

$$
\begin{aligned}
& M=\frac{a_{22}^{u} \bar{r}_{1}^{l}+a_{12}^{l} \bar{r}_{2}^{l}}{a_{11}^{u} a_{22}^{u}-a_{12}^{l} a_{21}^{l}} \triangleq \tilde{x}_{1}^{*}, \\
& N=\frac{a_{11}^{u} \bar{r}_{1}^{l}+a_{21}^{l} \bar{r}_{2}^{l}}{a_{11}^{u} a_{22}^{u}-a_{12}^{l} a_{21}^{l}} \triangleq \tilde{x}_{1}^{*} .
\end{aligned}
$$


Hence,

$$
\liminf _{t \rightarrow \infty} \frac{1}{t} \int_{0}^{t} x_{i}(s) d s \geq \widetilde{x}_{i}^{*}, \quad i=1,2, \text { a.s. }
$$

which is as required.

\section{Nonpersistence}

In this section, we discuss the dynamics of system (3) as the white noise is getting larger. We show that system (3) will be nonpersistent if the white noise is large, which does not happen in the deterministic system.

Definition 19. System (3) is said to be nonpersistent, if there are positive constants $q_{1}, q_{2}$ such that

$$
\lim _{t \rightarrow \infty} \prod_{i=1}^{2} x_{i}^{q_{i}}(t)=0 \quad \text { a.s. }
$$

Theorem 20. Assume that $a_{11}^{l} a_{22}^{l}>a_{12}^{u} a_{21}^{u}$ and $a_{22}^{l} \bar{r}_{1}^{u}+a_{12}^{u} \bar{r}_{2}^{u}<$ 0 , then system (3) is nompersistent, where $\bar{r}_{i}(s)=r_{i}(s)-$ $\left(\sigma_{i}^{2}(s) / 2\right), i=1,2$.

Proof. Since $x_{i}(t)>0, i=1,2$ and $a_{11}^{l} a_{22}^{l}>a_{12}^{u} a_{21}^{u}$, from (93) we have

$$
\begin{aligned}
& a_{22}^{l} \log x_{1}(t)+a_{12}^{u} \log x_{2}(t) \\
& \leq\left\{a_{22}^{l} \bar{r}_{1}^{u}+a_{12}^{u} \bar{r}_{2}^{u}\right\} t-\left(a_{11}^{l} a_{22}^{l}-a_{21}^{u} a_{12}^{u}\right) \int_{0}^{t} x_{1}(s) d s \\
& +a_{22}^{l}\left[\log x_{1}(0)+\int_{0}^{t} \sigma_{1}(s) d B_{1}(s)\right] \\
& +a_{12}^{u}\left[\log x_{2}(0)+\int_{0}^{t} \sigma_{2}(s) d B_{2}(s)\right] \\
& \leq K_{1} t+a_{22}^{l}\left[\log x_{1}(0)+\int_{0}^{t} \sigma_{1}(s) d B_{1}(s)\right] \\
& +a_{12}^{u}\left[\log x_{2}(0)+\int_{0}^{t} \sigma_{2}(s) d B_{2}(s)\right]
\end{aligned}
$$

where $K_{1}=a_{22}^{l} \bar{r}_{1}^{u}+a_{12}^{u} \bar{r}_{2}^{u}$ which together with

$$
\begin{aligned}
\lim _{t \rightarrow \infty} & \frac{a_{22}^{l}\left[\log x_{1}(0)+\int_{0}^{t} \sigma_{1}(s) d B_{1}(s)\right]}{t} \\
\quad & =\lim _{t \rightarrow \infty} \frac{a_{12}^{u}\left[\log x_{2}(0)+\int_{0}^{t} \sigma_{2}(s) d B_{2}(s)\right]}{t}=0, \quad \text { a.s., }
\end{aligned}
$$

implies

$$
\lim _{t \rightarrow \infty} \frac{1}{t}\left[a_{22}^{l} \log x_{1}(t)+a_{12}^{u} \log x_{2}(t)\right] \leq K_{1}, \quad \text { a.s. }
$$

If $K_{1}<0$, then there must be

$$
\lim _{t \rightarrow \infty} x_{1}^{a_{22}^{l}}(t) x_{2}^{a_{12}^{u}}(t)=0, \quad \text { a.s. }
$$

Hence, system (3) is nonpersistent.

Theorem 21. Assume that $a_{11}^{l} a_{22}^{l}>a_{12}^{u} a_{21}^{u}$ and $\left(a_{21}^{u} \bar{r}_{1}^{u}+\right.$ $\left.a_{11}^{l} \bar{r}_{2}^{u}\right)<0$, then system (3) is nonpersistent, where $\bar{r}_{i}(s)=$ $r_{i}(s)-\left(\sigma_{i}^{2}(s) / 2\right), i=1,2$.

Here we omit the proof of Theorem 21 which is similar to the proof of Theorem 20

Remark 22. If $\left(\sigma_{i}^{l}\right)^{2}>2 r_{i}^{u}, i=1,2$, then the conditions in Theorems 20 and 21 are obviously satisfied, respectively. That is to say, the large white noise will lead to the population system being non-persistent.

\section{Global Attractivity}

In this section, we turn to establishing sufficient criteria for the global attractivity of stochastic system (3).

Definition 23. Let $x(t), y(t)$ be two arbitrary solutions of system (3) with initial values $x(0), y(0) \in R_{+}^{2}$, respectively. If

$$
\lim _{t \rightarrow \infty}|x(t)-y(t)|=0, \quad \text { a.s., }
$$

then we say system (3) is globally attractive.

Theorem 24. Assume that $a_{11}^{l} a_{22}^{l}>a_{12}^{u} a_{21}^{u}$, then system (3) is globally attractive.

Proof. Let $x(t), y(t)$ be two arbitrary solutions of system (3) with initial values $x(0), y(0) \in R_{+}^{2}$. By the Itô's formula, we have

$$
\begin{aligned}
d \log x_{i}(t)= & {\left[r_{i}(t)-\frac{1}{2} \sigma_{i}^{2}(t)-a_{i i}(t) x_{i}(t)+a_{i j}(t) x_{j}(t)\right] d t } \\
& +\sigma_{i}(t) d B_{i}(t), \quad i, j=1,2, j \neq i,
\end{aligned}
$$

$$
\begin{aligned}
d \log y_{i}(t)= & {\left[r_{i}(t)-\frac{1}{2} \sigma_{i}^{2}(t)-a_{i i}(t) y_{i}(t)+a_{i j}(t) y_{j}(t)\right] d t } \\
& +\sigma_{i}(t) d B_{i}(t), \quad i, j=1,2, j \neq i .
\end{aligned}
$$

Then,

$$
\begin{array}{r}
d\left(\log x_{i}(t)-\log y_{i}(t)\right) \\
=\left\{-a_{i i}(t)\left[x_{i}(t)-y_{i}(t)\right]+a_{i j}(t)\left[x_{i}(t)-y_{i}(t)\right]\right\} d t, \\
i, j=1,2, j \neq i .
\end{array}
$$


Since $a_{11}^{l} a_{22}^{l}>a_{12}^{u} a_{21}^{u}$, there exist two positive constants $c_{1}, c_{2}$ which satisfy

$$
\frac{a_{21}^{u}}{a_{11}^{l}}<\frac{c_{1}}{c_{2}}<\frac{a_{22}^{l}}{a_{12}^{u}} .
$$

Thus, $c_{1} a_{11}^{l}-c_{2} a_{21}^{u}>0, c_{2} a_{22}^{l}-c_{1} a_{12}^{u}>0$.

Consider a Lyapunov function $V(t)$ defined by

$$
\begin{aligned}
V(t)= & c_{1}\left|\log x_{1}(t)-\log y_{1}(t)\right| \\
& +c_{2}\left|\log x_{2}(t)-\log y_{2}(t)\right|, \quad t \geq 0 .
\end{aligned}
$$

A direct calculation of the right differential $d^{+} V(t)$ of $V(t)$ along the ordinary differential equation (119) leads to

$$
\begin{aligned}
d^{+} V(t)= & c_{1} \operatorname{sgn}\left(x_{1}(t)-y_{1}(t)\right) d\left[\log x_{1}(t)-\log y_{1}(t)\right] \\
& +c_{2} \operatorname{sgn}\left(x_{2}(t)-y_{2}(t)\right) d\left[\log x_{2}(t)-\log y_{2}(t)\right] \\
= & c_{1} \operatorname{sgn}\left(x_{1}(t)-y_{1}(t)\right) \\
& \times\left[-a_{11}(t)\left(x_{1}(t)-y_{1}(t)\right) d t\right. \\
& \left.+a_{12}(t)\left(x_{2}(t)-y_{2}(t)\right) d t\right] \\
& +c_{2} \operatorname{sgn}\left(x_{2}(t)-y_{2}(t)\right) \\
& \times\left[a_{21}(t)\left(x_{1}(t)-y_{1}(t)\right) d t\right. \\
& \left.-a_{22}(t)\left(x_{2}(t)-y_{2}(t)\right) d t\right] \\
\leq & -c_{1} a_{11}^{l}\left|x_{1}(t)-y_{1}(t)\right| d t \\
& +c_{1} a_{12}^{u}\left|x_{2}(t)-y_{2}(t)\right| d t \\
& -c_{2} a_{22}^{l}\left|x_{2}(t)-y_{2}(t)\right| d t \\
& +c_{2} a_{21}^{u}\left|x_{1}(t)-y_{1}(t)\right| d t \\
= & -\left(c_{1} a_{11}^{l}-c_{2} a_{21}^{u}\right)\left|x_{1}(t)-y_{1}(t)\right| d t \\
& -\left(c_{2} a_{22}^{l}-c_{1} a_{12}^{u}\right)\left|x_{2}(t)-y_{2}(t)\right| d t \\
\leq & -\gamma_{i=1}^{2}\left|x_{i}(t)-y_{i}(t)\right| d t \\
&
\end{aligned}
$$

where $\gamma=\min \left\{c_{1} a_{11}^{l}-c_{2} a_{21}^{u}, c_{2} a_{22}^{l}-c_{1} a_{12}^{u}\right\}$. Integrating both sides of (122) form 0 to $t$, we have

$$
V(t)+\gamma \int_{0}^{t} \sum_{i=1}^{2}\left|x_{i}(s)-y_{i}(s)\right| d s \leq V(0)<\infty .
$$

Let $t \rightarrow \infty$, we obtain

$$
\begin{aligned}
\int_{0}^{\infty}|x(s)-y(s)| d s & \leq \int_{0}^{\infty} \sum_{i=1}^{2}\left|x_{i}(s)-y_{i}(s)\right| d s \\
& \leq \frac{V(0)}{\gamma}<\infty \quad \text { a.s. }
\end{aligned}
$$

Note that $u(t)=x(t)-y(t)$. Clearly, $u(t) \in C\left(R_{+}, R^{2}\right)$ a.s. It is straightforward to see from (124) that

$$
\liminf _{t \rightarrow \infty}|u(t)|=0 \quad \text { a.s. }
$$

Next, we prove that

$$
\lim _{t \rightarrow \infty}|u(t)|=0 \quad \text { a.s. }
$$

By Theorem 3 we obtain that the pth moment of the solution of system (3) is bounded, the following proof is similar to the proof of Theorem 6.2 in [15] and hence is omitted.

\section{Acknowledgments}

The work was supported by the Ph.D. Programs Foundation of Ministry of China (no. 200918), NSFC of China (no. 10971021), and Program for Changjiang Scholars and Innovative Research Team in University.

\section{References}

[1] R. Redheffer, "Nonautonomous Lotka-Volterra systems. I," Journal of Differential Equations, vol. 127, no. 2, pp. 519-541, 1996.

[2] R. Redheffer, "Nonautonomous Lotka-Volterra systems. II," Journal of Differential Equations, vol. 132, no. 1, pp. 1-20, 1996.

[3] A. Tineo, "On the asymptotic behavior of some population models. II," Journal of Mathematical Analysis and Applications, vol. 197, no. 1, pp. 249-258, 1996.

[4] Z. D. Teng, "Nonautonomous Lotka-Volterra systems with delays," Journal of Differential Equations, vol. 179, no. 2, pp. 538561, 2002.

[5] F. Y. Wei and W. Ke, "Global stability and asymptotically periodic solution for nonautonomous cooperative Lotka-Volterra diffusion system," Applied Mathematics and Computation, vol. 182, no. 1, pp. 161-165, 2006.

[6] Y. Nakata and Y. Muroya, "Permanence for nonautonomous Lotka-Volterra cooperative systems with delays," Nonlinear Analysis. Real World Applications, vol. 11, no. 1, pp. 528-534, 2010.

[7] X. Abdurahman and Z. Teng, "On the persistence of a nonautonomous $n$-species Lotka-Volterra cooperative system," Applied Mathematics and Computation, vol. 152, no. 3, pp. 885895, 2004.

[8] C. Y. Ji, D. Q. Jiang, H. Liu, and Q. S. Yang, "Existence, uniqueness and ergodicity of positive solution of mutualism system with stochastic perturbation," Mathematical Problems in Engineering, vol. 2010, Article ID 684926, 18 pages, 2010.

[9] C. Y. Ji and D. Q. Jiang, "Persistence and non-persistence of a mutualism system with stochastic perturbation," Discrete and Continuous Dynamical Systems A, vol. 32, no. 3, pp. 867-889, 2012.

[10] X. R. Mao, S. Sabanis, and E. Renshaw, "Asymptotic behaviour of the stochastic Lotka-Volterra model," Journal of Mathematical Analysis and Applications, vol. 287, no. 1, pp. 141-156, 2003.

[11] X. R. Mao, C. G. Yuan, and J. Z. Zou, "Stochastic differential delay equations of population dynamics," Journal of Mathematical Analysis and Applications, vol. 304, no. 1, pp. 296-320, 2005.

[12] M. Liu and K. Wang, "Survival analysis of a stochastic cooperation system in a polluted environment," Journal of Biological Systems, vol. 19, no. 2, pp. 183-204, 2011. 
[13] D. Q. Jiang and N. Z. Shi, "A note on nonautonomous logistic equation with random perturbation," Journal of Mathematical Analysis and Applications, vol. 303, no. 1, pp. 164-172, 2005.

[14] D. Q. Jiang, N. Z. Shi, and X. Y. Li, “Global stability and stochastic permanence of a non-autonomous logistic equation with random perturbation," Journal of Mathematical Analysis and Applications, vol. 340, no. 1, pp. 588-597, 2008.

[15] X. Y. Li and X. R. Mao, "Population dynamical behavior of nonautonomous Lotka-Volterra competitive system with random perturbation," Discrete and Continuous Dynamical Systems A, vol. 24, no. 2, pp. 523-545, 2009.

[16] M. Liu and K. Wang, "Persistence and extinction in stochastic non-autonomous logistic systems," Journal of Mathematical Analysis and Applications, vol. 375, no. 2, pp. 443-457, 2011.

[17] M. Liu and K. Wang, "Asymptotic properties and simulations of a stochastic logistic model under regime switching II," Mathematical and Computer Modelling, vol. 55, no. 3-4, pp. 405418, 2012.

[18] X. R. Mao, Stochastic Differential Equations and Applications, Horwood, Chichester, UK, 1997.

[19] X. Mao, G. Marion, and E. Renshaw, "Environmental Brownian noise suppresses explosions in population dynamics," Stochastic Processes and their Applications, vol. 97, no. 1, pp. 95-110, 2002.

[20] I. N. Wantanabe, Stochastic Differential Equations and Diffusion Processes, North-Holland, Amsterdam, The Netherlands, 1981.

[21] H. P. Liu and Z. E. Ma, "The threshold of survival for system of two species in a polluted environment," Journal of Mathematical Biology, vol. 30, no. 1, pp. 49-61, 1991. 


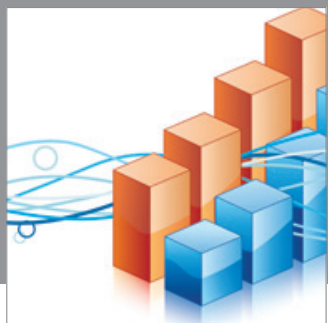

Advances in

Operations Research

mansans

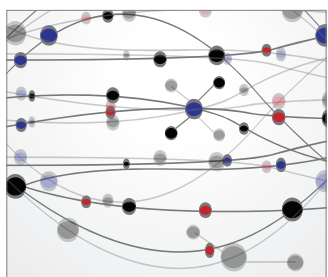

The Scientific World Journal
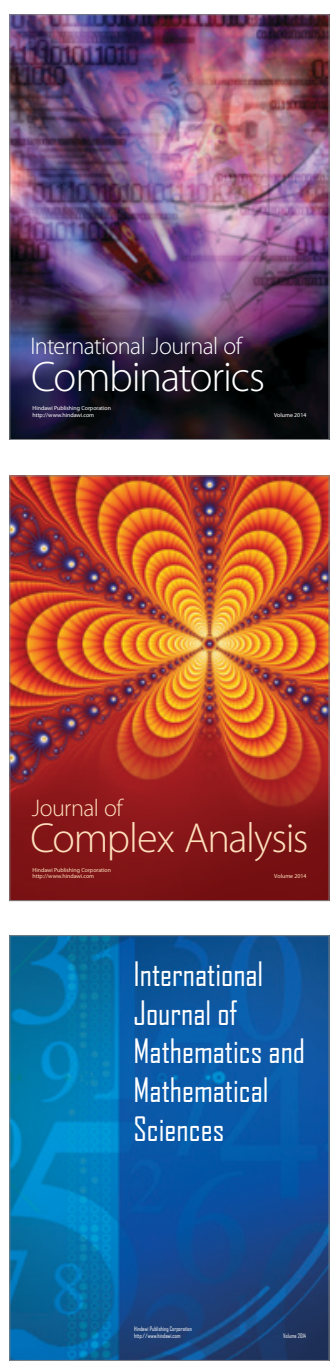
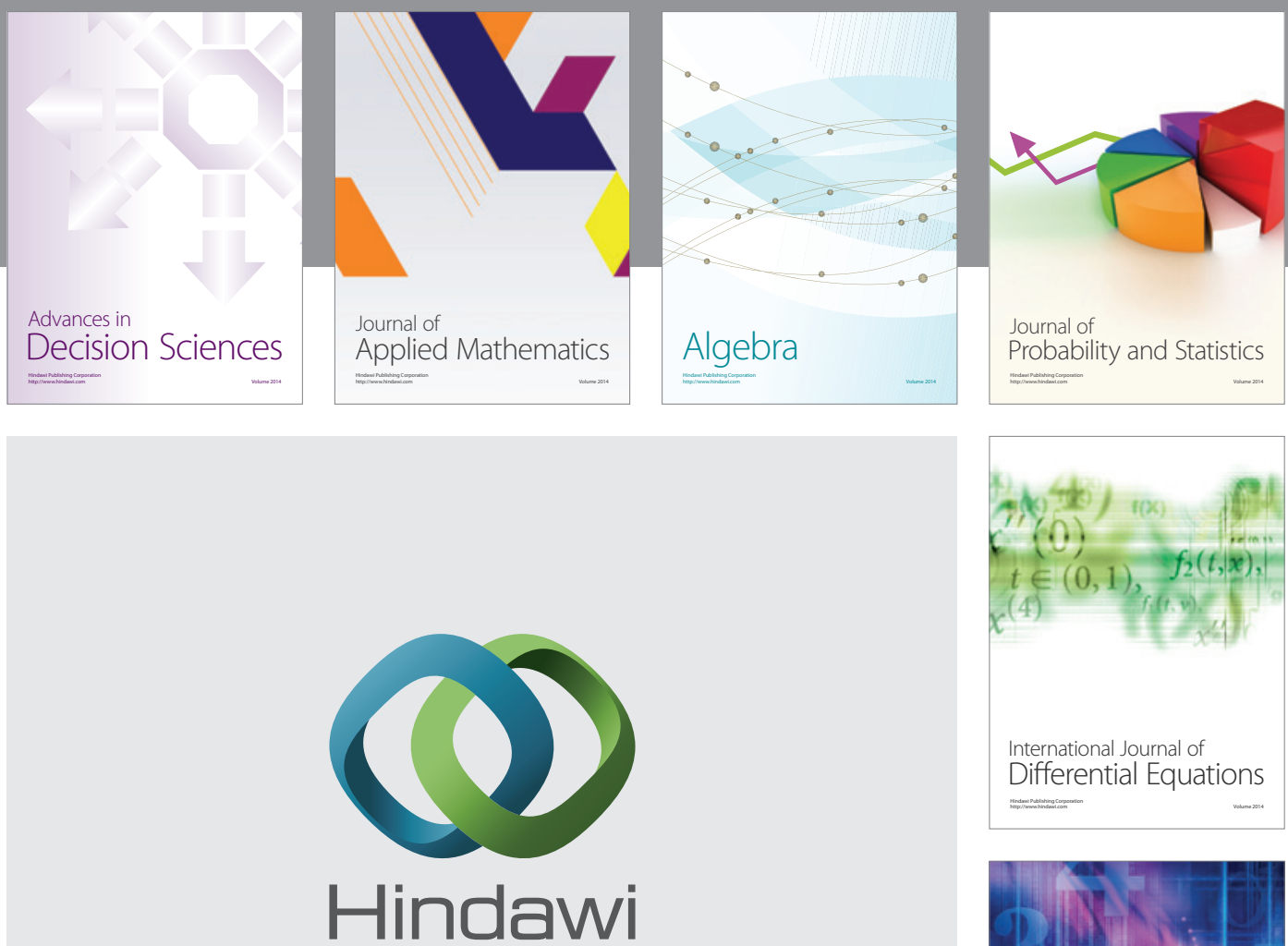

Submit your manuscripts at http://www.hindawi.com
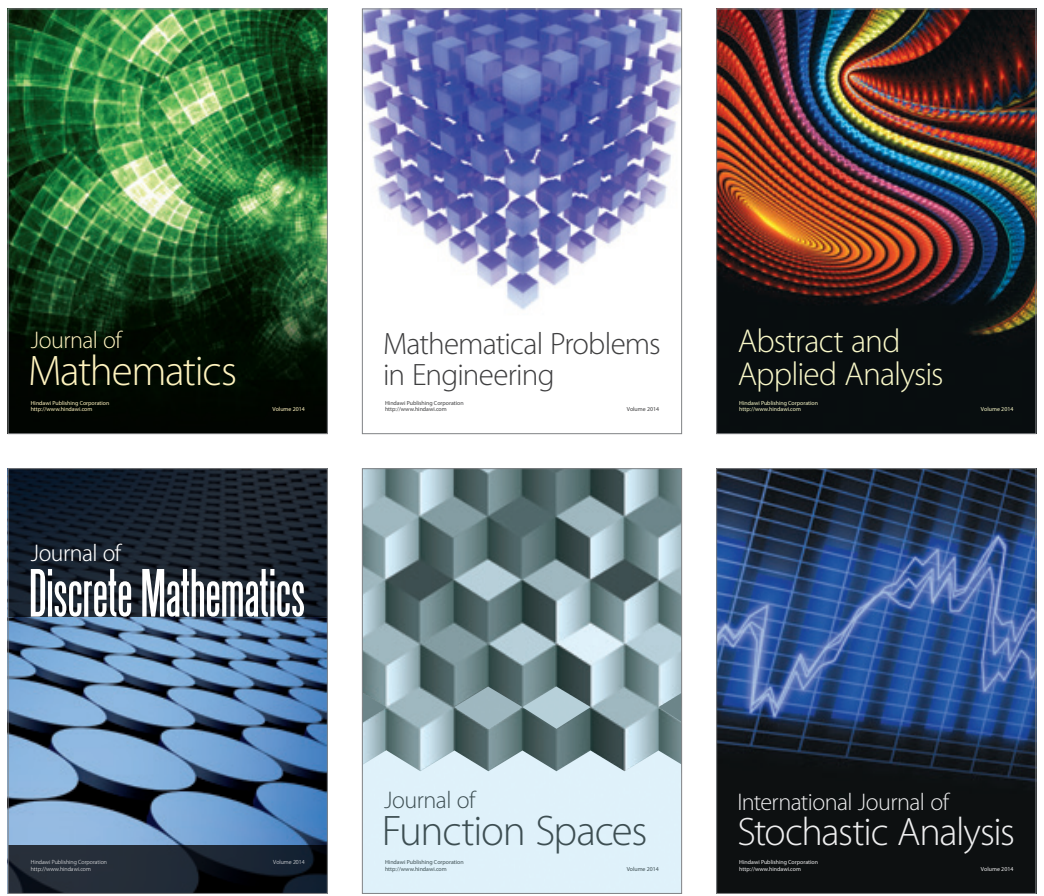

Journal of

Function Spaces

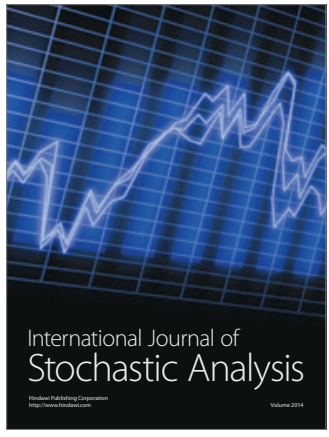

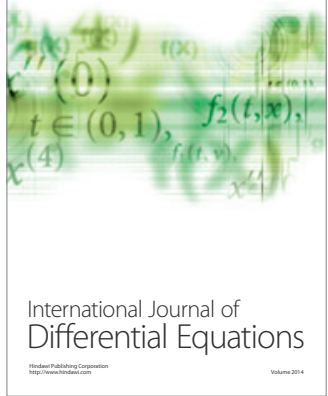
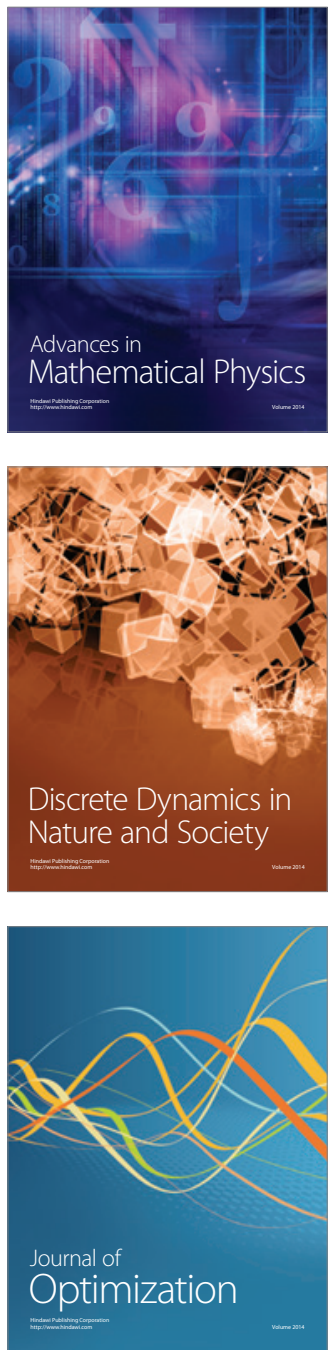